\title{
IMPLICATIONS OF PLANCK AND MAP MEASUREMENTS ON SPARTICLE SPECTRA
}

\author{
R. ARNOWITT \\ Center for Theoretical Physics, Department of Physics, \\ Texas AEMM University, College Station, TX 77843-4242, USA \\ PRAN NATH \\ Department of Physics, Northeastern University, Boston, MA 02115, USA
}

\begin{abstract}
Future sattelite (MAP and Planck), balloon and ground based experiments will determine the basic cosmological parameters within a few percent. We examine here the effect of this on constraining the SUSY parameter space for supergravity $R$-parity conserving models (with $\tan \beta \leq 25$ ) for the cases of $\nu \mathrm{CDM}$ and $\Lambda \mathrm{CDM}$ cosmological models. For the $\nu \mathrm{CDM}(\Lambda \mathrm{CDM})$ models, the gluino mass is restricted by $m_{\tilde{g}} \lesssim 720(540) \mathrm{GeV}$. In both cases, the cosmological constraints are sensitive to non-universal SUSY soft breaking producing a lower bound $m_{\tilde{g}}>400 \mathrm{GeV}$ in some regions and for the $\nu \mathrm{CDM}$ model, gaps in the allowed $m_{\tilde{g}}$ range for other regions. For gluino (neutralino) masses greater than $450(65) \mathrm{GeV}, m_{0}$ is constrained to be small making squark and slepton masses generally light and determined mostly by $m_{\tilde{g}}$.
\end{abstract}

\section{Introduction}

One of the significant phenomena that has arisen with the development of supersymmetry has been the deepening of the connection between particle physics and cosmology. While this connection has always been present (e.g. from the early nuclear synthesis calculations 6 ) supersymmetry (SUSY) now offers models that reach up in energy to the GUT scale $M_{G} \approx 10^{16} \mathrm{GeV}$ (and perhaps further to the Planck scale) and backwards in time to the very early universe. In particular, models with $R$-parity invariance automatically predict the existence of dark matter, and for a resonable range of SUSY parameters, in an amount comparable to what is observed astronomically. These models have been waiting to be tested, and there are now a large number of experiments that are currently on line or will be on line in the relatively near future that will determine whether supersymmetry is a valid theory of nature.

While up to now the flow of information has been mostly from particle physics to cosmology, the new sattelite experiments 3 MAP and Planck as well as about 25 balloon and ground based experiments can reverse this and can give constraints on what might be expected at accelerators. These astronomical experiments will measure the cosmological parameters with great accuracy. One uses the parameter $\Omega_{i}=\rho_{i} / \rho_{c}$ where $\rho_{i}$ is the density of matter of type 
$i$ and $\rho_{c}=3 H^{2} / 8 \pi G_{N}$ ( $H=$ Hubble constant, $G_{N}=$ Newton's constant $)$ to measure the mean amount of matter of type $i$ in the universe. Current mea-

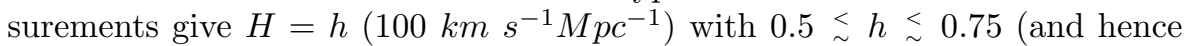
$\left.\rho_{c}=1.88 \times 10^{-29} h^{2} \mathrm{gm} / \mathrm{cm}^{3}\right)$. For a number of cosmological models one finds for cold dark matter $(\mathrm{CDM})$ the range

$$
0.1 \leq \Omega_{C D M} h^{2} \leq 0.4
$$

and this range of CDM is the one assumed in many particle physics calculations. However, MAP and Planck measurements will greatly restrict this window, and impose strong constraints on the SUSY parameter space, and in this way restrict the SUSY mass spectrum. Thus astronomical measurements will impinge upon what is expected to be seen at accelerators.

\section{Supergravity Models}

In order to analyse dark matter predictions we need a well-defined SUSY model. We will use here supergravity grand unified models with gravity mediated SUSY breaking (at a scale $\sim M_{G}$ ) in a hidden sector, and with gravity being the messenger of SUSY breaking to the physical sector 3 . This gives rise to the following types of soft breaking masses at $M_{G}$ 3. breaking mass), $m_{1 / 2}$ (gaugino soft breaking mass), $A_{0}$ (cubic soft breaking parameter), $B_{0}$ (quadratic soft breaking parameter), as well as non-universal soft

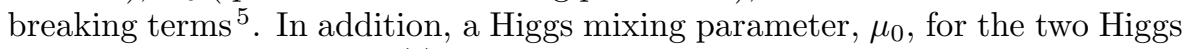
multiplets $H_{1}$ and $H_{2}\left(W^{(2)}=\mu_{0} H_{1} H_{2}\right)$ can form naturally with $\mu_{0} \approx M_{S}$.

In the following we will assume that the gaugino masses are universal at $M_{G}$ (as would be true to a good approximation for a simple GUT group). Over much of the parameter space $\mu^{2} \gg M_{Z}^{2}$ which leads to the "scaling relations" for the charginos $\left(\chi_{i}^{ \pm}, i=1,2\right)$ and neutralinos $\left(\chi_{i}^{0}, i=1-4\right) \mathrm{d}$ : $2 m_{\chi_{1}^{0}} \cong m_{\chi_{1}^{ \pm}} \cong m_{\chi_{2}^{0}} \cong\left(\frac{1}{3}-\frac{1}{4}\right) m_{\tilde{g}}$.

We assume here that the first two generations of squarks and sleptons are degenerate at $M_{G}$ (to suppress flavor changing neutral currents) with common mass $m_{0}$ and parametrize the Higgs and third generation soft breaking masses at $M_{G}$ as:

$$
\begin{gathered}
m_{H_{1}}^{2}=m_{0}^{2}\left(1+\delta_{1}\right) ; m_{H_{2}}^{2}=m_{0}^{2}\left(1+\delta_{2}\right) \\
m_{q_{L}}^{2}=m_{0}^{2}\left(1+\delta_{3}\right) ; m_{u_{R}}^{2}=m_{0}^{2}\left(1+\delta_{4}\right) ; m_{e_{R}}^{2}=m_{0}^{2}\left(1+\delta_{5}\right) \\
m_{d_{R}}^{2}=m_{0}^{2}\left(1+\delta_{6}\right) ; m_{l_{L}}^{2}=m_{0}^{2}\left(1+\delta_{7}\right)
\end{gathered}
$$

Here $q_{L} \equiv\left(\tilde{u}_{L}, \tilde{d}_{L}\right)$ is the $L$ squark doublet, $u_{R}$ the $R$ up-squark singlet, etc. We limit our parameters to the domain $m_{0}, m_{\tilde{g}} \leq 1 \mathrm{TeV} ;\left|A_{t} / m_{0}\right| \leq$ 
$7 ; \tan \beta \leq 25 ;\left|\delta_{i}\right| \leq 1$ where $A_{t}$ is the $t$-quark $A$-parameter at the electroweak scale. These represent a choice of "naturalness" conditions. Note that for $\tan \beta \lesssim 25, \delta_{5}, \delta_{6}, \delta_{7}$ make only small contributions and can be neglected. (They would become important for larger $\tan \beta$.)

The RGE then give for $\mu^{2}$ at scale $M_{Z}$ the result 6 :

$$
\begin{aligned}
\mu^{2}= & \frac{t^{2}}{t^{2}-1}\left[\left\{\frac{1-3 D_{0}}{2}+\frac{1}{t^{2}}\right\}+\left\{\frac{1-D_{0}}{2}\left(\delta_{3}+\delta_{4}\right)-\frac{1+D_{0}}{2} \delta_{2}+\frac{1}{t^{2}} \delta_{1}\right\}\right] m_{0}^{2} \\
& +\frac{t^{2}}{t^{2}-1}\left[\frac{1}{2}\left(1-D_{0}\right) \frac{A_{R}^{2}}{D_{0}}+C_{\tilde{g}} m_{\tilde{g}}^{2}\right]-\frac{1}{2} M_{Z}^{2} \\
& +\frac{1}{22} \frac{t^{2}+1}{t^{2}-1}\left(1+\frac{\alpha_{1}}{\alpha_{G}}\right) S_{0}+\text { loop terms } ; \quad t \equiv \tan \beta
\end{aligned}
$$

Here $D_{0} \cong 1-\left(m_{t} / 200 \sin \beta\right)^{2}, A_{R} \cong A_{t}-0.613 m_{\tilde{g}}, S_{0}=\operatorname{Tr} Y m^{2}(Y=$ hypercharge, $m^{2}=$ masses at $M_{G}$ ) and $C_{\tilde{g}}$ is given in Ibañez et al $D_{0}$ vanishes at the $t$ - quark Landau pole and hence for $m_{t}=175 \mathrm{GeV}$ is generally small: $D_{0} \leq 0.23$. ( $A_{R}$ is the residue at the Landau pole.) Note that the choice $\delta_{2}<0, \delta_{1}>0$ and $\delta_{3}+\delta_{4}>0$ will increase the size of $\mu^{2}$, while $\delta_{2}>0, \delta_{1}<0$ and $\delta_{3}+\delta_{4}<0$ will decrease the size of $\mu^{2}$. In the following we will see that $\mu^{2}$ plays a key role in dark matter predictions, and the effects of these particular sign possibilities of $\delta_{i}$ will allow a qualitative understanding of the phenomena.

\section{Direct Detection of Dark Matter}

We review briefly in this section the direct detection of dark matter particles in the Milky Way incident on a terrestial detector. The density of such matter is estimated at $\rho_{D M} \cong 0.3 \mathrm{GeV} / \mathrm{cm}^{3}$ with an impinging velocity of $v_{D M} \cong$ $300 \mathrm{~km} / \mathrm{s}$. Calculation of detector event rates proceeds in two steps. One first calculates the expected $\chi_{1}^{0}$ relic density of $\Omega_{\chi_{1}^{0}} h^{2}$, left after annihilation in the early universe 6 . The size of $\Omega_{\chi_{1}^{0}} h^{2}$ varies as one moves across the SUSY parameter space. In particular, for $m_{\chi_{1}^{0}} \sim 65 \mathrm{GeV}$ (or by the scaling relations,

$m_{\tilde{g}} \sim 450 \mathrm{GeV}$ ) the annihilation cross section is dominated by the $s$-channel $Z$ and $h$ poles and requires sensitive treatment 10.11 . For $m_{\chi_{1}^{0}}>65 \mathrm{GeV}$, the $t$-channel squark and slepton poles become dominant. These two regimes will show up below in the detector event rates.

One first restricts the SUSY parameter space so that $\Omega_{\chi_{1}^{0}} h^{2}$ falls within the allowed window of the cosmological model under consideration. In addition, one restricts the parameter space so that accelerator bounds are obeyed. One then calculates the scattering of incident Milky Way $\chi_{1}^{0}$ by quarks in a nuclear 
target $\mathrm{Q}$ within the above restricted parameter space, giving rise to detector event rates $R$ measured in events $/ \mathrm{kg} d$.

\section{Cosmological Models}

Current astronomical measurements have sufficient uncertainty that they allow for a large variety of cosmological models. However, the Planck and MAP sattelites will be able to determine the basic cosmological parameters, $H, \Lambda, \Omega$ etc. to (1-10)\% accuracy by measurements of the deviations $\Delta T$ from the CMB temperature $T_{0}=(2.728 \pm 0.002){ }^{\circ} \mathrm{K}$. Different cosmological models predict different angular correlations of the CMB deviations allowing for experimental determination of the cosmological model. We consider here two possible cosmological models that might result from such measurements, and examine what consequences these might have on the sparticle spectra, and hence on accelerator searches for supersymmetry.

(i) $\nu C D M$ Model. If neutrinos have masses of $O(e V)$ they could furnish the hot dark matter (HDM) of the universe, $\Omega_{\nu}$. In addition there is baryonic dark matter $(B)$ and $\mathrm{CDM}$ (the neutralinos $\chi_{1}^{0}$ ) such that the total $\Omega=1$. As an example we assume that the sattelite measurements determine central values to be $\Omega_{\nu}=0.2, \Omega_{B}=0.05, \Omega_{C D M}=0.75$ and a Hubble constant of $h=0.62$. Using estimates of the errors with which these quantities can be measured by the Planck sattelite 13 , t4 we find

$$
\Omega_{C D M} h^{2}=0.288 \pm 0.013
$$

This shows the remarkable accuracy future determinations of cosmological parameters are capable of when compared with the current knowledge of Eq. (11).

(ii) $\Lambda C D M$ Model. As a second model we assume the existence of a cosmological constant $\Lambda$ along with baryonic and CDM, and assume that Planck has determined the central values of these parameters to be $\Omega_{B}=0.05$, $\Omega_{C D M}=0.40, \Omega_{\Lambda}=0.55$ and $h=0.62$. Using the estimates of the errors in the Planck measurements 13 one finds here that

$$
\Omega_{C D M} h^{2}=0.154 \pm 0.017
$$

While the above models are only two possible examples, they represent bounds on what might actually exist. The important point is that the future balloon and sattelite measurements will be able to determine these parameters well, and the above models represent limits within current knowledge. 
$\nu \operatorname{CDM}(1 \sigma), \delta_{1}=0=\delta_{2}$

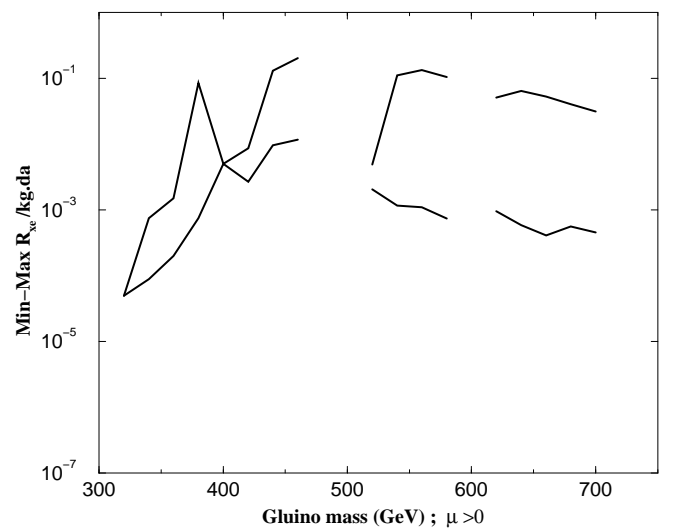

Figure 1: Maximum and minimum event rates for a xenon CDM detector vs gluino mass for $\nu \mathrm{CDM}$ model (1 std window) for universal soft breaking, $\mu>0$. From Ref 16 .

\section{$5 \quad \nu$ CDM Model}

We consider first universal soft breaking where by Eqs. (2)-(4), the $\delta_{i}=0$. Fig. 1 plots the maximim and minimum event rates for Xe detector as a function of the gluino mass $\left[m_{\tilde{g}} \cong(7-8) m_{\chi_{1}^{0}}\right.$ by the scaling relations] for a 1 std band of Eq. (6). One may compare this with Fig. 2 of 3 where only the broad band of Eq. (1) is imposed. One sees a reduction in event rates in the region $m_{\tilde{g}} \lesssim 450 \mathrm{GeV}$ (i.e. $m_{\chi_{1}^{0}}<65 \mathrm{GeV}$ ), the $Z$ and $h$ pole dominated region in the early universe annihilation, and a corresponding increase for the higher masses. More significant is the appearance of forbidden regions, i.e. gaps, in the allowed values of $m_{\tilde{g}}$ in the region $m_{\tilde{g}} \cong 500 \mathrm{GeV}$ and $m_{\tilde{g}} \cong 600 \mathrm{GeV}$. In examining the effects of non-universal soft breaking, we note the correlation that when $\mu^{2}$ is decreased (which by Eq. (5) occurs for $\delta_{2}=1=-\delta_{1}$ ) the event rate $R$ is increased and when $\mu^{2}$ is increased $\left(\delta_{2}=-1=-\delta_{1}\right) R$ is decreased. Fig. 2 shows these effects for the latter case, and also that the forbidden regions below $m_{\tilde{g}}=500 \mathrm{GeV}$ are significantly widened. For the alternate possibility $\left(\delta_{2}=1=-\delta_{1}\right)$, the event rates increase. The maximum rates for this case are at the sensitivity of the current $\mathrm{NaI}$ detectors 15 , and detectors with a sensitivity of $R>10^{-3}$ events $/ \mathrm{kg} d$ would cover the entire parameter space. The gaps of the previous case have now disappeared. However, $m_{\tilde{g}}$ is now bounded from below i.e. $m_{\tilde{g}} \sim 420 \mathrm{GeV}$. In all of these cases there is 
$\operatorname{vCDM}(1 \sigma), \delta_{1}=1=-\delta_{2}$

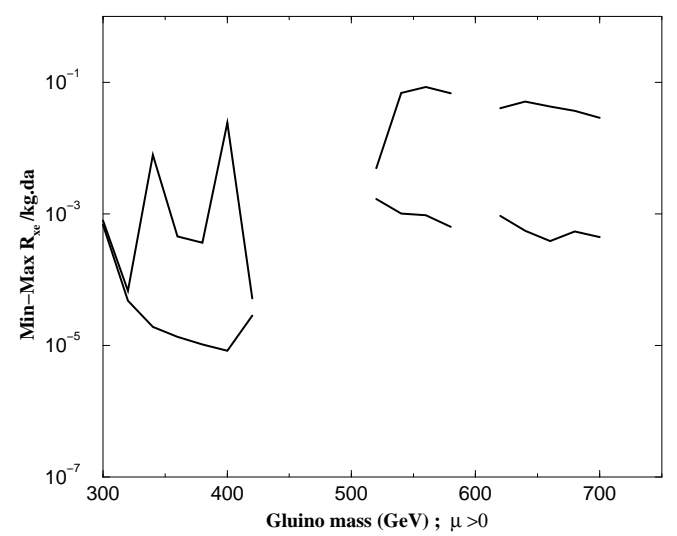

Figure 2: Same as Fig.1 for non-universal soft breaking with $\delta_{1}=1=-\delta_{2}$. From Ref 16 .

also an upper bound on $m_{\tilde{g}}$ of $m_{\tilde{g}} \stackrel{\sim}{\sim} 720 \mathrm{GeV}$. Thus cosmological constraints bound the allowed gluino mass range, and are sensitive to the non-universal soft breaking.

The large $t$-quark mass causes large $L-R$ mixing in the stop (mass) ${ }^{2}$ matrix, making the light eigenvalue, $m_{\tilde{t}_{1}}^{2}$, small. In general $m_{\tilde{t}_{1}}$ is governed by the size of $m_{0}$ and in the light neutralino domain $m_{0}$ can be large, i.e. $m_{\tilde{t}_{1}}$ can rise to $\approx 600 \mathrm{GeV}$. However, for heavy neutralinos $\left(m_{\tilde{Z}_{1}} \geq 65 \mathrm{GeV}\right.$, $m_{\tilde{g}} \gtrsim 450 \mathrm{GeV}$ ) the early neutralino annihilation is governed by the $t$-channel sfermion poles, and in order to get sufficient annihilation for Eq. (6) to hold, $m_{0}$ must be small i.e. $m_{0} \lesssim 200 \mathrm{GeV}$. One finds in this domain that $m_{\tilde{t}_{1}}$ is generally an increasing function of $m_{\tilde{g}}$ with mass ranging from $250 \mathrm{GeV}$ to $500 \mathrm{GeV}$.

\section{$6 \quad \Lambda$ CDM Model}

Here $\Omega_{\chi_{1}^{0}} h^{2}$ obeys Eq. (7) which even more sharply restricts the SUSY parameter space. Fig. 3 shows the maximum and minimum expected event rates for a Xe detector for different values of $\delta_{i}$. While one has the same general behavior as in the $\nu \mathrm{CDM}$ model, there are interesting differences that distinguish the two. Thus for this case for the 1 std window of Eq. (7), one finds a much tighter upper bound of $m_{\tilde{g}} \sim 520 \mathrm{GeV}$. There is also a lower bound $m_{\tilde{g}} \gtrsim 400 \mathrm{GeV}$ for $\delta_{2}=1=-\delta_{1}$ (dashed curve), sharply restricting the gluino 


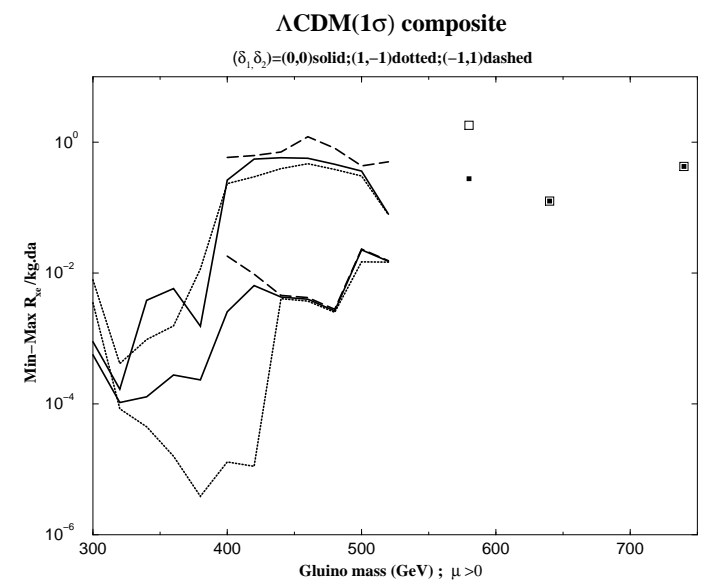

Figure 3: Maximum and minimum event rates for a xenon CDM detector vs. gluino for $\Lambda \mathrm{CDM}$ model (1 std window), $\mu>0$, for universal soft breaking (solid curve) and nonuniversal soft breaking $\delta_{1}=1=-\delta_{2}$ (dotted curve), $\delta_{1}=-1=-\delta_{2}$ (dashed curve). (The discrete high mass points are for $\delta_{1}=-1=-\delta_{2}$ where scaling is badly broken.) From Ref 16.

mass range for this case. Also, there are no gaps in the allowed gluino mass range.

The squarks and sleptons are again constrained to be relatively light in the domain $m_{\tilde{g}}>420 \mathrm{GeV}$ since here Eq. (7) requires $m_{0} \stackrel{\sim}{\sim} 100 \mathrm{GeV}$. Thus $m_{\tilde{t}_{1}}$ ranges from $(200-400) \mathrm{GeV}$ in this domain and for the first two generations of squarks, $m_{\tilde{q}}$ ranges from $400 \mathrm{GeV}$ to $500 \mathrm{GeV}$ and approximately scales with $m_{\tilde{g}}$. The lightest slepton, $\tilde{e}_{R}$ can have a mass as low as $(85-90) \mathrm{GeV}$ in this region, at the edge of detectability of LEP 190.

This work was supported in part by National Science Foundation Grants PHY-9722090 and PHY-96020274.

\section{References}

1. E. M. Burbidge, G. R. Burbidge, W. A. Fowler and F. Hoyle, Rev. Mod. Phys. 29, 547 (1957); P. J. E. Peebles, Ast. J. 146, 542 (1966); R. V. Wagoner, W. A. Fowler and F. Hoyle, Ap. J. 148, 3 (1967).

2. http://map.gsfc.nasa.gov/: http://astro.estec.esa.nl:80/SA-general/Projects/Cobras/cobras.html.

3. A. H. Chamseddine, R. Arnowitt and P. Nath, Phys. Rev. Lett. 49, 
970 (1982). For reviews see P. Nath, R. Arnowitt and A. H. Chamseddine, Applied N=1 Supergravity (World Scientific, Singapore, 1984); H. P. Nilles, Phys. Rep. 110, 1 (1984); R. Arnowitt and P. Nath, Proc. of VII J.A. Swieca Summer School ed. E. Eboli and V. O. Rivelles (World Scientific, Singapore, 1994).

4. R. Barbieri, S. Ferrara and C. A. Savoy, Phys. Lett. B 119, 343 (1982); L. Hall, J. Lykken and S. Weinberg, Phys. Rev. D 27, 2359 (1983); P. Nath, R. Arnowitt and A. H. Chamseddine, Nucl. Phys. B 227, 121 (1983).

5. S. K. Soni and H. A. Weldon, Phys. Lett. B 126, 215 (1983); V. S. Kaplunovsky and J. Louis, Phys. Lett. B 306, 268 (1993).

6. R. Arnowitt and P. Nath, Phys. Rev. Lett. 69, 725 (1992). P. Nath and R. Arnowitt, Phys. Lett. B 289, 368 (1992).

7. K. Inoue et al. Theor. Phys. 68, 927 (1982); L. Ibañez and G. G. Ross, Phys. Lett. B 110, 227 (1982); L. Alvarez-Gaumé, J. Polchinski and M. B. Wise, Nucl. Phys. B 221, 495 (1983); J. Ellis, J. Hagelin, D. V. Nanopoulos and K. Tamvakis, Phys. Lett. B 125, 2275 (1983); L. E. Ibañez and C. Lopez, Nucl. Phys. B 233, 545 (1984); L.E. Ibañez, C. Lopez and C. Muños, Nucl. Phys. B 256, 218 (1985).

8. P. Nath and R. Arnowitt, Phys. Rev. D 56, 2820 (1997).

9. For a review see G. Jungman, M. Kamionkowski and K. Greist, Phys. Rep. 267, 195 (1995); E. W. Kolb and M.S. Turner, The Early Universe (Addison-Wesley, Reading, 1989).

10. K. Greist and D. Seckel, Phys. Rev. D 43, 3191 (1991); P. Gondolo and G. Gelmini, Nucl. Phys. B 360, 145 (1991).

11. R. Arnowitt and P. Nath, Phys. Lett. B 299, 58 (1993); 303, 403 (1993) (E); P. Nath and R. Arnowitt, Phys. Rev. Lett. 70, 3696 (1993); H. Baer and M. Brhlick, Phys. Rev. D 53, 597 (1996); V. Barger and C. Kao, hep-ph/9704403.

12. For a review see Ref.[9].

13. A. Kosowsky, M. Kamionkowski, G. Jungman and D. Spergel, Nucl. Phys. Proc. Suppl. 51B, 49 (1996).

14. S. Dodelson, E. Gates and A. Stebbins, Ap. J. 467, 10 (1996).

15. A. Bottino et al, Phys. Lett. B 402, 113 (1997).

16. P. Nath and R. Arnowitt, hep-ph/9801xxx. 